\title{
IMAGING THE MOLECULAR GAS PROPERTIES OF A MAJOR MERGER DRIVING THE EVOLUTION OF A $z=2.5$ SUBMILLIMETER GALAXY
}

\author{
Dominik A. Riechers ${ }^{1,8}$, Christopher L. Carilli ${ }^{2}$, Fabian Walter $^{3}$, Axel Weiss $^{4}$, JefF WagG $^{5}$, Frank Bertoldi $^{6}$, \\ Dennis Downes ${ }^{7}$, Christian Henkel ${ }^{4}$, and Jacqueline Hodge ${ }^{3}$ \\ ${ }^{1}$ Astronomy Department, California Institute of Technology, MC 249-17, 1200 East California Boulevard, Pasadena, CA 91125, USA; dr@ caltech.edu \\ ${ }^{2}$ National Radio Astronomy Observatory, P.O. Box O, Socorro, NM 87801, USA \\ ${ }^{3}$ Max-Planck-Institut für Astronomie, Königstuhl 17, D-69117 Heidelberg, Germany \\ ${ }^{4}$ Max-Planck-Institut für Radioastronomie, Auf dem Hügel 69, Bonn, D-53121, Germany \\ ${ }^{5}$ European Southern Observatory, Alonso de Córdova 3107, Vitacura, Casilla 19001, Santiago 19, Chile \\ ${ }^{6}$ Argelander-Institut für Astronomie, Universität Bonn, Auf dem Hügel 71, Bonn, D-53121, Germany \\ ${ }^{7}$ Institut de RadioAstronomie Millimétrique, 300 Rue de la Piscine, Domaine Universitaire, 38406 Saint Martin d'Héres, France \\ Received 2010 September 7; accepted 2011 April 12; published 2011 May 2
}

\begin{abstract}
We report the detection of spatially extended $\mathrm{CO}(J=1 \rightarrow 0)$ and $\mathrm{CO}(J=5 \rightarrow 4)$ emission in the $z=2.49$ submillimeter galaxy (SMG) J123707+6214, using the Expanded Very Large Array and the Plateau de Bure Interferometer. The large molecular gas reservoir is spatially resolved into two $\mathrm{CO}(J=1 \rightarrow 0)$ components (northeast and southwest; previously identified in $\mathrm{CO} J=3 \rightarrow 2$ emission) with respective gas masses of 4.3 and $3.5 \times 10^{10}\left(\alpha_{\mathrm{CO}} / 0.8\right) M_{\odot}$. We thus find that the optically invisible northeast component slightly dominates the gas mass in this system. The total molecular gas mass derived from the $\mathrm{CO}(J=1 \rightarrow 0)$ observations is $\gtrsim 2.5 \times$ larger than estimated from $\mathrm{CO}(J=3 \rightarrow 2)$. The two components are at approximately the same redshift, but separated by $\sim 20 \mathrm{kpc}$ in projection. The morphology is consistent with that of an early-stage merger. The total amount of molecular gas is sufficient to maintain the intense $500 M_{\odot} \mathrm{yr}^{-1}$ starburst in this system for at least $\sim 160 \mathrm{Myr}$. We derive line brightness temperature ratios of $r_{31}=0.39 \pm 0.09$ and $0.37 \pm 0.10$, and $r_{51}=0.26 \pm 0.07$ and $0.25 \pm 0.08$ in the two components, respectively, suggesting that the $J \geqslant 3$ lines are substantially subthermally excited. This also suggests comparable conditions for star formation in both components. Given the similar gas masses of both components, this is consistent with the comparable starburst strengths observed in the radio continuum emission. Our findings are consistent with other recent studies that find evidence for lower CO excitation in SMGs than in high- $z$ quasar host galaxies with comparable gas masses. This may provide supporting evidence that both populations correspond to different evolutionary stages in the formation of massive galaxies.
\end{abstract}

Key words: cosmology: observations - galaxies: active - galaxies: formation - galaxies: high-redshift - galaxies: starburst - radio lines: galaxies

Online-only material: color figures

\section{INTRODUCTION}

Detailed studies of submillimeter galaxies (SMGs; see review by Blain et al. 2002) have revealed that they represent a relatively rare, but cosmologically important high-redshift population of massive galaxies. They harbor intense $\left(>500 M_{\odot} \mathrm{yr}^{-1}\right)$, often heavily obscured, but rather short-lived ( $<100 \mathrm{Myr})$ starbursts that rapidly consume their gas content through star formation at high efficiencies. SMGs may trace a common phase in the formation and evolution of massive galaxies in the early universe, making them the likely progenitors of today's massive spheroidal galaxies.

Given their substantial dust obscuration, the most insightful way to study SMGs and their star formation properties is through the dust-reprocessed emission at rest-frame far-infrared (FIR) wavelengths. While continuum diagnostics at such wavelengths are particularly useful to determine star formation rates (SFRs) unaffected by obscuration, the most insightful way to study the fate of such galaxies is through emission line fluxes, morphology, and dynamics of the material that fuels the star formation, i.e., molecular gas (typically CO). Molecular gas was detected in $>30$ SMGs to date, revealing large gas reservoirs

\footnotetext{
8 Hubble Fellow
}

of $>10^{10} M_{\odot}$ in most cases (see Solomon \& Vanden Bout 2005 for a review). However, most of these studies were carried out in mid- to high- $J$ CO transitions, rather than the fundamental $\mathrm{CO}(J=1 \rightarrow 0)$ transition.

Recent studies of $\mathrm{CO}(J=1 \rightarrow 0)$ emission indicate that (in contrast to high- $z$ quasar hosts) this line appears to carry a higher brightness temperature than the mid- to high- $J$ lines in several SMGs, suggesting relatively low-excitation gas excitation (e.g., Hainline et al. 2006; Riechers et al. 2006, 2010; Carilli et al. 2010; Ivison et al. 2010; Harris et al. 2010). Therefore, earlier studies may systematically underestimate the total amount of molecular gas that is present in SMGs. The low excitation in at least part of the molecular gas reservoirs raises the question whether the commonly used $\alpha_{\mathrm{CO}}$ conversion factor from $\mathrm{CO}$ luminosity to gas mass $\left(M_{\mathrm{gas}}\right)$ for ultra-luminous infrared galaxies (ULIRGs) in the nearby universe (Downes \& Solomon 1998) is applicable, or if this practice leads to an underprediction of $M_{\text {gas }}$.

To further investigate this issue, we have started a systematic Expanded Very Large Array (EVLA) survey of $\mathrm{CO}(J=1 \rightarrow 0)$ emission in high- $z$ SMGs and quasar host galaxies that were previously detected in higher- $J$ CO lines. In this Letter, we report the first results from this study, i.e., the detection of spatially extended $\mathrm{CO}(J=1 \rightarrow 0)$ emission toward the $z=2.49$ 
SMG J123707+6214 (GN19; HDF242; Borys et al. 2003). We also report the detection of $\mathrm{CO}(J=5 \rightarrow 4)$ emission in this system, using the Plateau de Bure Interferometer (PdBI). We use a concordance, flat $\Lambda \mathrm{CDM}$ cosmology throughout, with $H_{0}=71 \mathrm{~km} \mathrm{~s}^{-1} \mathrm{Mpc}^{-1}, \Omega_{\mathrm{M}}=0.27$, and $\Omega_{\Lambda}=0.73$ (Spergel et al. 2003, 2007).

\section{OBSERVATIONS}

\subsection{EVLA}

We observed the $\mathrm{CO}(J=1 \rightarrow 0)\left(v_{\text {rest }}=115.2712 \mathrm{GHz}\right)$ emission line toward J123707+6214 using the EVLA. At $z=2.49$, this line is redshifted to $33.029 \mathrm{GHz}(9.08 \mathrm{~mm})$. Observations were carried out under excellent weather conditions (typical atmospheric phase rms: 2.3 on a $300 \mathrm{~m}$ baseline) in D array on 2010 April 11 (NRAO Legacy ID: AR708), resulting in $2.9 \mathrm{hr}$ on-source time with 18 antennas (equivalent to $1.2 \mathrm{hr}$ with 27 antennas) after rejection of bad data. The nearby (5.4 distance) quasar J1302+5748 was observed every 7.5 minutes for pointing, secondary amplitude, and phase calibration. For primary flux calibration, the standard calibrator 3C286 was observed, leading to a calibration that is accurate within $\lesssim 10 \%$. Observations were set up using a total bandwidth of $252 \mathrm{MHz}$ (dual polarization; after rejection of overlapping edge channels between sub-bands; corresponding to $\sim 2300 \mathrm{~km} \mathrm{~s}^{-1}$ at $9.08 \mathrm{~mm}$ ) with the WIDAR correlator.

For data reduction and analysis, the AIPS package was used. All data were mapped using "natural" weighting. Maps of the velocity-integrated $\mathrm{CO} J=1 \rightarrow 0$ line emission yield a synthesized clean beam size of $2^{\prime \prime} .8 \times 2$ '. 1 at an rms noise level of $39 \mu \mathrm{Jy} \mathrm{beam}^{-1}$ over $490 \mathrm{~km} \mathrm{~s}^{-1}(54 \mathrm{MHz})$.

\section{2. $P d B I$}

We observed the $\mathrm{CO}(J=5 \rightarrow 4)\left(v_{\text {rest }}=576.2679 \mathrm{GHz}\right)$ emission line toward J123707+6214 using the IRAM PdBI. At $z=2.49$, this line is redshifted to $165.1198 \mathrm{GHz}(1.82 \mathrm{~mm})$. Observations were carried out under good weather conditions with five antennas in the compact $\mathrm{D}$ configuration during three tracks on 2008 July 10, and August 9 and 14 (IRAM program ID: SC47), for a total of $14.9 \mathrm{hr}$, resulting in $8.1 \mathrm{hr}$ of six antenna equivalent on-source time after rejection of bad data. The nearby quasars B0954+658 and B1418+546 (distance to J123707+6214: 17.4 and 15.4) were observed every 20 minutes for pointing, secondary amplitude, and phase calibration. For primary flux calibration, the standard calibrators MWC 349 and 3C454.3 were observed. Observations were set up using a total spectrometer bandwidth of $1 \mathrm{GHz}$ (dual polarization; corresponding to $\sim 1800 \mathrm{~km} \mathrm{~s}^{-1}$ at $1.82 \mathrm{~mm}$ ).

For data reduction and analysis, the GILDAS package was used. All data were mapped using "natural" weighting. Maps of the velocity-integrated $\mathrm{CO} J=5 \rightarrow 4$ line emission yield a synthesized clean beam size of 3 .' $7 \times 3$ ". 0 at an rms noise level of $0.39 / 0.54 \mathrm{mJy} \mathrm{beam}^{-1}$ over $563 / 309 \mathrm{~km} \mathrm{~s}^{-1}(320 / 180 \mathrm{MHz})$.

\section{RESULTS}

\subsection{Gas Morphology and Emission Line Properties}

We have detected spatially resolved $\mathrm{CO}(J=1 \rightarrow 0)$ line emission toward the $z=2.49$ SMG J123707+6214, measuring two components at $7 \sigma$ (northeast, "ne") ${ }^{9}$ and $6 \sigma$ (southwest,

\footnotetext{
9 Following the nomenclature by Tacconi et al. (2006).
}

"sw") significance in the velocity-integrated emission line map (Figure 1, left). We do not detect the underlying continuum emission at a $3 \sigma$ upper limit of $54 \mu \mathrm{Jy} \mathrm{beam}^{-1}$ at $9.08 \mathrm{~mm}$ (rest-frame $2.6 \mathrm{~mm}$ ).

From Gaussian fitting to the $\mathrm{CO}(J=1 \rightarrow 0)$ line profiles of the ne and sw components (Figure 1 , right), ${ }^{10}$ we obtain line peak strengths of $S_{v}=375 \pm 57$ and $340 \pm 64 \mu \mathrm{Jy}$ at line FWHMs of $d v=454 \pm 87$ and $414 \pm 92 \mathrm{~km} \mathrm{~s}^{-1}$, centered at redshifts of $z=2.4879 \pm 0.0005$ and $2.4873 \pm 0.0005$, respectively. The line widths and redshifts are consistent with those measured for the $\mathrm{CO}(J=3 \rightarrow 2)$ emission line (Tacconi et al. 2006), and are equal for both spatial components within the uncertainties. From the spatially integrated emission, we determine a systemic redshift of $z=2.4876 \pm 0.0004$, which we adopt as the nominal value for the system in the following. The line parameters for J123707+6214ne and sw correspond to velocity-integrated emission line strengths of $I_{\mathrm{CO}(1-0)}=$ $0.180 \pm 0.029$ and $0.149 \pm 0.029 \mathrm{Jy} \mathrm{km} \mathrm{s}^{-1}$, i.e., line luminosities of $L_{\mathrm{CO}(1-0)}^{\prime}=(5.36 \pm 0.86)$ and $(4.43 \pm$ $0.85) \times 10^{10} \mathrm{~K} \mathrm{~km} \mathrm{~s}^{-1} \mathrm{pc}^{2}$.

We have also detected spatially resolved $\mathrm{CO}(J=5 \rightarrow 4)$ line emission at $\gtrsim 5 \sigma$ significance toward both components of J123707+6214 (Figure 2). The ne component dominates the integrated line emission (Figure 2, left), extracted over a velocity range comparable to the $\mathrm{CO}(J=1 \rightarrow 0)$ map. The maximum signal-to-noise ratio on the sw component is obtained over a narrower velocity range (Figure 2, middle). In this map, the sw component is brighter than the ne component, comparable to what is seen in the $\mathrm{CO}(J=3 \rightarrow 2)$ maps of Tacconi et al. (2006, 2008 ). Only $\sim 60 \%$ of the emission from the ne component are seen over this narrower velocity range. We do not detect the underlying continuum emission at a $3 \sigma$ upper limit of $0.8 \mathrm{mJy}$ beam $^{-1}$ at $1.82 \mathrm{~mm}$ (rest-frame $520 \mu \mathrm{m}$ ).

From Gaussian fitting to the integrated $\mathrm{CO}(J=5 \rightarrow 4)$ line profile (Figure 2, right), we obtain $S_{v}=4.1 \pm 0.8 \mathrm{mJy}$ at $d v=485 \pm 110 \mathrm{~km} \mathrm{~s}^{-1}$, centered at $z=2.4875 \pm 0.0006$. This corresponds to $I_{\mathrm{CO}(5-4)}=2.12 \pm 0.51 \mathrm{Jy} \mathrm{km} \mathrm{s}^{-1}$. Fitting the ne and sw components individually yields $S_{v}=2.4 \pm 0.5$ and $2.1 \pm 0.5 \mathrm{mJy}, d v=467 \pm 124$ and $432 \pm 130 \mathrm{~km} \mathrm{~s}^{-1}$, and $I_{\mathrm{CO}(5-4)}=1.17 \pm 0.33$ and $0.94 \pm 0.29 \mathrm{Jy} \mathrm{km} \mathrm{s}^{-1}$, respectively. We thus derive $L_{\mathrm{CO}(5-4)}^{\prime}=(1.39 \pm 0.32)$ and $(1.12 \pm$ $0.29) \times 10^{10} \mathrm{~K} \mathrm{~km} \mathrm{~s}^{-1} \mathrm{pc}^{2}$, respectively.

This implies $\mathrm{CO} J=3 \rightarrow 2 / 1 \rightarrow 0$ line brightness temperature ratios of $r_{31}=0.39 \pm 0.09(\text { ne) })^{11}$ and $0.37 \pm 0.10(\mathrm{sw})$, and $\mathrm{CO} J=5 \rightarrow 4 / 1 \rightarrow 0$ line brightness temperature ratios of $r_{51}=0.26 \pm 0.07$ and $0.25 \pm 0.08$. The $\mathrm{CO}(J=3 \rightarrow 2)$ and $\mathrm{CO}(J=5 \rightarrow 4)$ emission lines are clearly subthermally excited toward both components $\left(r_{31}<1\right.$ and $\left.r_{51}<1\right)$. Interestingly, both components appear to have comparable gas excitation. The ne component is brighter in all $\mathrm{CO}$ transitions. This suggests that the ne component carries the dominant fraction of the molecular gas mass in this system. Based on a ULIRG conversion factor $\alpha_{\mathrm{CO}}=0.8 M_{\odot}\left(\mathrm{K} \mathrm{km} \mathrm{s}^{-1} \mathrm{pc}^{2}\right)^{-1}$ to derive $M_{\mathrm{gas}}$ from $L_{\mathrm{CO}(1-0)}^{\prime}$ (Downes \& Solomon 1998), we determine the total molecular gas masses of $\mathrm{J} 123707+6214 \mathrm{ne}$ and sw to be $M_{\mathrm{gas}}=4.3$ and

\footnotetext{
$10 \mathrm{CO}(J=1 \rightarrow 0)$ spectra are Hanning-smoothed.

11 We recomputed the $\operatorname{CO}(J=3 \rightarrow 2)$ fluxes based on the data presented in Tacconi et al. (2006) by extracting the emission over the same (broader) velocity range as the $\mathrm{CO}(J=1 \rightarrow 0)$ and $\mathrm{CO}(J=5 \rightarrow 4)$ emission. This yields $I_{\mathrm{CO}(3-2)}=0.63 \pm 0.10$ and $0.50 \pm 0.10 \mathrm{Jy} \mathrm{km} \mathrm{s}^{-1}$ for the ne and sw components, respectively. The different methods for extracting fluxes are likely responsible for differences between our analysis and an independent $\mathrm{CO}(J=1 \rightarrow 0)$ study carried out in parallel by Ivison et al. (2011).
} 

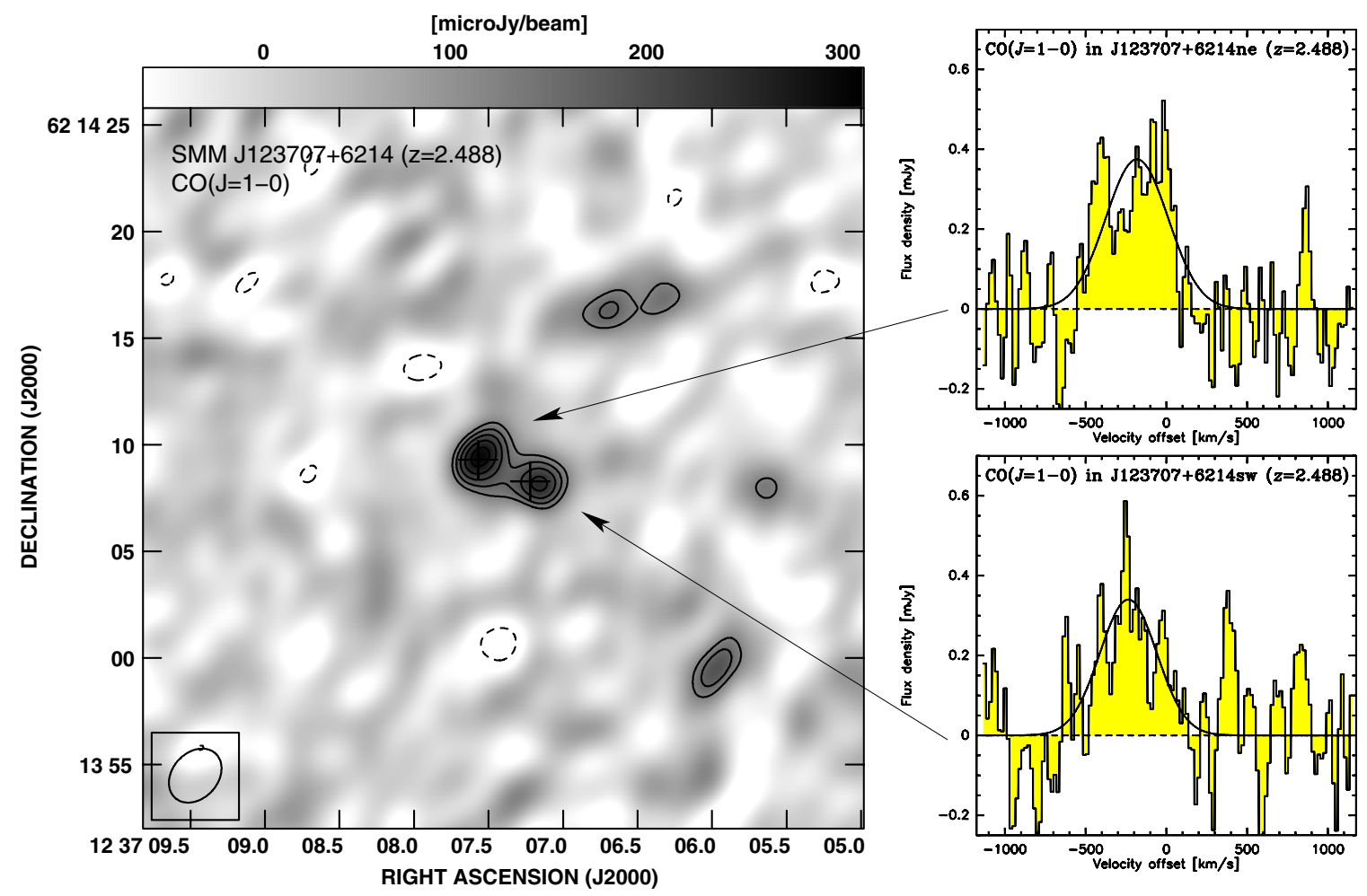

Figure 1. EVLA $\operatorname{CO}(J=1 \rightarrow 0)$ emission toward $\mathrm{J} 123707+6214$. Left: velocity-integrated $\mathrm{CO}(J=1 \rightarrow 0)$ map over $490 \mathrm{~km} \mathrm{~s}^{-1}(54 \mathrm{MHz})$. At a resolution of 2 ". $8 \times 2$ ". 1 (as indicated in the bottom left), the emission is resolved into a northeast (ne) and southwest (sw) component. The crosses indicate the peak positions of the $\mathrm{CO}(J=$ $3 \rightarrow 2$ ) emission (Tacconi et al. 2006). Contours are shown in steps of $1 \sigma=39 \mu \mathrm{Jy} \mathrm{beam}^{-1}$, starting at $\pm 3 \sigma$. Right: beam-corrected $\mathrm{CO}(J=1 \rightarrow 0)$ spectra toward the ne (top) and sw (bottom) components at $18 \mathrm{~km} \mathrm{~s}^{-1}$ ( $2 \mathrm{MHz}$ ) resolution (histograms), along with Gaussian fits to the line emission (black curves). The velocity scale is relative to the tuning frequency of $33.029 \mathrm{GHz}$ (corresponding to $z=2.49$ ).

(A color version of this figure is available in the online journal.)
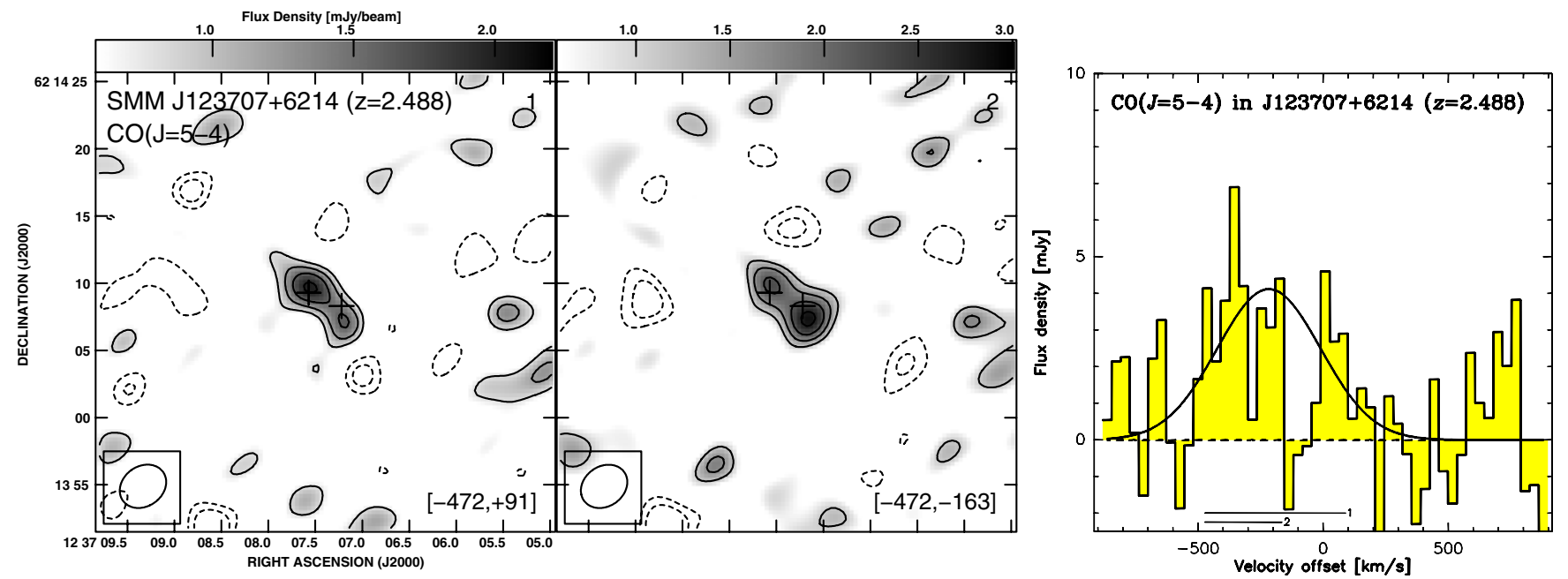

Figure 2. PdBI $\mathrm{CO}(J=5 \rightarrow 4)$ emission toward $\mathrm{J} 123707+6214$. Left/middle: velocity-integrated $\mathrm{CO}(J=5 \rightarrow 4)$ maps over $563 / 309 \mathrm{~km} \mathrm{~s}{ }^{-1}(320 / 180 \mathrm{MHz})$. At a resolution of $3^{\prime \prime} .7 \times 3^{\prime \prime} .0$ (as indicated in the bottom left), the emission is resolved into the ne and sw components also $\operatorname{seen}$ in $\operatorname{CO}(J=1 \rightarrow 0)$. The velocity ranges are selected to maximize the integrated (left) and sw component (middle) signal-to-noise ratio. The crosses are the same as in Figure 1. Contours are shown in steps of $1 \sigma=0.39 / 0.54 \mathrm{mJy}_{\text {beam }}^{-1}$, starting at $\pm 2 \sigma$. Right: integrated $\mathrm{CO}(J=5 \rightarrow 4)$ spectrum at $36 \mathrm{~km} \mathrm{~s}^{-1}(20 \mathrm{MHz})$ resolution (histogram), along with a Gaussian fit to the line emission (black curve). The velocity ranges of the two maps are indicated by the black lines. The velocity scale is relative to the tuning frequency of $165.1198 \mathrm{GHz}$ (corresponding to $z=2.49$ ).

(A color version of this figure is available in the online journal.)

$3.5 \times 10^{10} M_{\odot},{ }^{12}$ i.e., by more than a factor of two higher than previously found based on the $\mathrm{CO}(J=3 \rightarrow 2)$ data (scaled to the same $\alpha_{\mathrm{CO}}$ ), and corresponding to $\sim 2 / 3$ of the stellar mass in this system (Tacconi et al. 2006, 2008).

12 A Milky Way like $\alpha_{\mathrm{CO}}=3.5 M_{\odot}\left(\mathrm{K} \mathrm{km} \mathrm{s}^{-1} \mathrm{pc}^{2}\right)^{-1}$ (e.g., Daddi et al. 2010) would increase $M_{\text {gas }}$ by a factor of 4.4 .

\subsection{Dynamical Structure of the Gas Reservoir}

In Figure 3, maps of the $\operatorname{CO}(J=1 \rightarrow 0)$ emission are shown in $182 \mathrm{~km} \mathrm{~s}^{-1}$ wide velocity channels. The emission toward J123707+6214ne appears dynamically resolved on $\sim 1$ 1.5 $(\sim 12 \mathrm{kpc})$ scales, which may suggest that the emission is more spatially extended than in the $\mathrm{CO}(J=3 \rightarrow 2)$ line $\left(0^{\prime} \cdot 5 \pm 0,2\right.$, or 


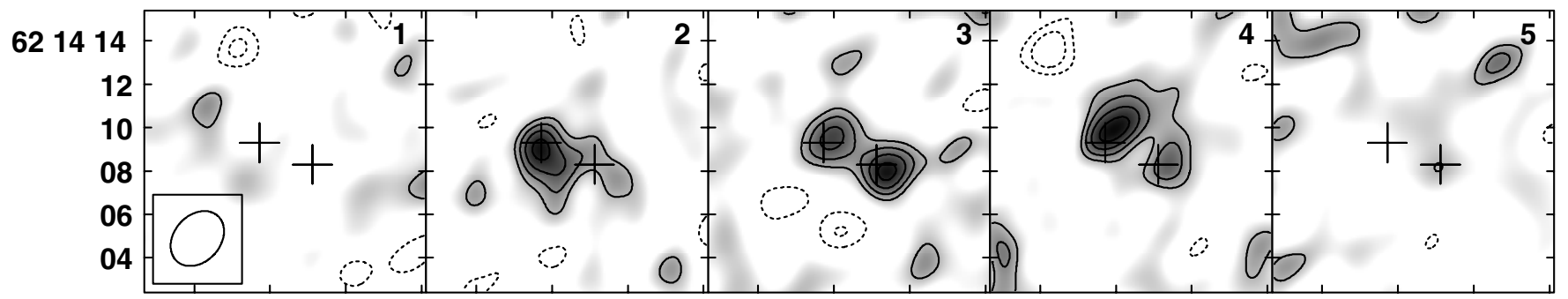

$123708.0 \quad 07.5 \quad 07.0 \quad 06.5$

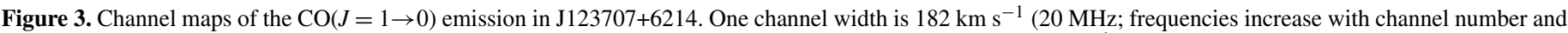

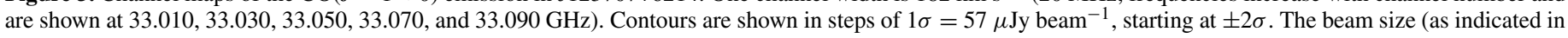
the bottom left) and crosses are the same as in Figure 1.

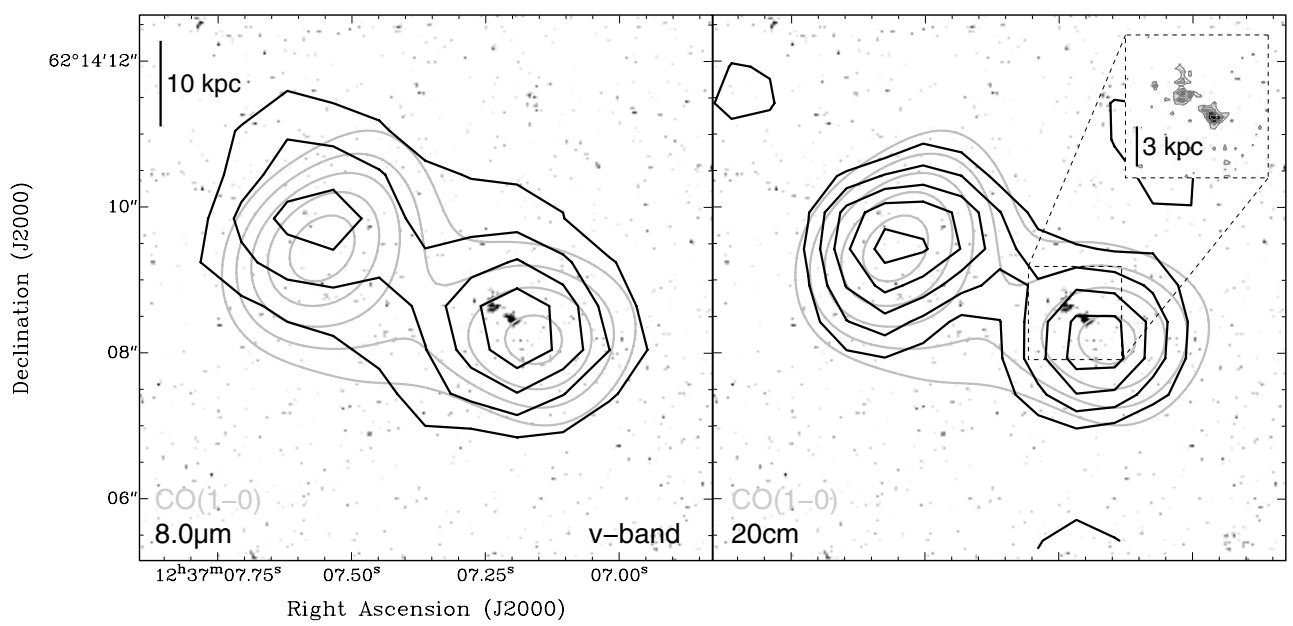

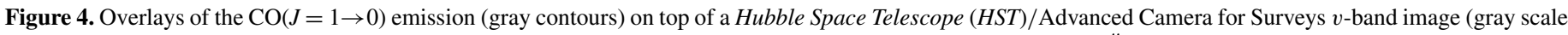

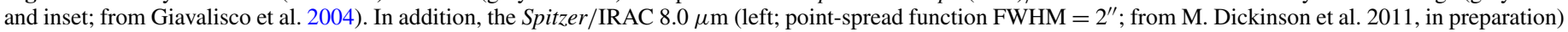
and VLA $20 \mathrm{~cm}$ (right; 1". $7 \times$ 1.' 6 resolution; from Morrison et al. 2010) emission are shown (black contours in each panel).

$4.1 \pm 1.6 \mathrm{kpc}$; Tacconi et al. 2006). J123707+6214sw appears marginally spatially resolved in position-velocity space at best, consistent with the size measured in $\operatorname{CO}(J=3 \rightarrow 2)$ emission within the relative uncertainties $(0.9 \pm 0.3$, or $7.4 \pm 2.5 \mathrm{kpc}$; Tacconi et al. 2006). Assuming radii of 6 and $3.7 \mathrm{kpc}$ for $\mathrm{J} 123707+6214 \mathrm{ne}$ and sw, this yields dynamical masses of $M_{\mathrm{dyn}}$ $\sin ^{2} i=2.9$ and $1.5 \times 10^{11} M_{\odot}$ (which we estimate to be reliable within a factor of two). This is about twice as high as previous estimates based on $\mathrm{CO}(J=3 \rightarrow 2$ ) emission (Tacconi et al. 2008), and corresponds to gas mass fractions of $f_{\text {gas }}=0.15$ and 0.23 for $\mathrm{J} 123707+6214 \mathrm{ne}$ and $\mathrm{sw}$, respectively. Higher resolution observations are required to better constrain how the merger dynamics impact the $\mathrm{CO}$ line profiles and the morphology of the gas reservoir, which is necessary to determine more precise dynamical masses.

\section{ANALYSIS AND DISCUSSION}

\subsection{Origin of the $\mathrm{CO}$ Emission}

SMGs are commonly associated with heavily obscured starbursts. J123707+6214 is a particularly insightful example of this population, as the ne component remains undetected at all wavelengths shortward of $3.6 \mu \mathrm{m}$ (rest-frame $1.0 \mu \mathrm{m}$ ). As shown in Figure 4 , the peak of the $\operatorname{CO}(J=1 \rightarrow 0)$ emission of the ne component is clearly associated with peaks in the mid-infrared $(8.0 \mu \mathrm{m}$; rest-frame $2.3 \mu \mathrm{m})$ and radio continuum $(20 \mathrm{~cm}$; restframe $6 \mathrm{~cm}$; see also Tacconi et al. 2008). The ne component also slightly dominates the radio emission $(\sim 55 \%)$, which suggests that it contributes the dominant fraction to the source's SFR. As also shown in Figure 4, J123707+6214sw consists of multiple components in the optical (606 nm; rest-frame $174 \mathrm{~nm})$ that are separated by a few kpc (see also Swinbank et al. 2004). This may correspond to multiple star-forming clumps, embedded in a more complex, extended molecular gas reservoir, but may also reflect the high degree of obscuration in this source.

\subsection{Gas Surface Densities and Star Formation Timescales}

Estimating that the $\mathrm{CO}(J=1 \rightarrow 0)$ emission in $\mathrm{J} 123707+$ $6214 \mathrm{ne}$ and sw is distributed over 6 and $3.7 \mathrm{kpc}$ radius regions, the surface-averaged gas densities are $\Sigma_{\text {gas }}=3.8$ and $8.1 \times 10^{8} M_{\odot} \mathrm{kpc}^{-2}$, i.e., comparable to but somewhat lower than estimates based on the (assuming the above size estimates) more compact $\mathrm{CO}(J=3 \rightarrow 2)$ emission (Tacconi et al. 2006, 2008). This would be consistent with some of the $\mathrm{CO}(J=1 \rightarrow 0)$ emission being in a diffuse, low surface brightness component.

Based on the SFR of $500 \pm 250 M_{\odot} \mathrm{yr}^{-1}$ determined by Tacconi et al. (2008), we derive a gas depletion timescale of $\tau_{\text {dep }}=M_{\text {gas }} /$ SFR $\sim 160$ Myr for J123707+6214. This is consistent with but on the high end of what is found for other SMGs (for which $M_{\text {gas }}$ are inferred from mid-J CO lines; e.g., Greve et al. 2005).

\section{CONCLUSIONS}

We have detected spatially resolved $\mathrm{CO}(J=1 \rightarrow 0)$ and $\mathrm{CO}(J=5 \rightarrow 4)$ emission toward the $z=2.49 \mathrm{SMG}$ $\mathrm{J} 123707+6214$. We resolve the emission into two components previously detected in $\mathrm{CO}(J=3 \rightarrow 2$ ) emission (Tacconi et al. 
2006, 2008), which are likely merging galaxies. Both components show similar $\mathrm{CO}$ excitation properties, with moderate $J=3 \rightarrow 2 / 1 \rightarrow 0$ line ratios of $r_{31} \sim 0.38$ and relatively low $J=5 \rightarrow 4 / 1 \rightarrow 0$ line ratios of $r_{51} \sim 0.25$. The implied $J=5 \rightarrow 4 / 3 \rightarrow 2$ line ratios of $r_{53} \sim 0.66$ are comparable to those found in other SMGs that show evidence for mergers (e.g., Weiß et al. 2005). On the other hand, the low $r_{31}$ are comparable to those found in massive gas-rich star-forming galaxies with much lower SFRs (Dannerbauer et al. 2009; Aravena et al. 2010). This may suggest that, in addition to the highly excited gas associated with the starburst, J123707+6214 hosts a substantial amount of low-excitation gas.

The $\mathrm{CO}(J=1 \rightarrow 0)$ emission suggests the presence of $\gtrsim 2.5 \times$ more molecular gas than expected if assuming a constant brightness temperature from $\mathrm{CO}(J=3 \rightarrow 2)$. The optically detected merger component (sw) carries $\sim 45 \%$ of the gas mass in this system, suggesting comparable amounts of gas in both components, with a slightly higher contribution coming from the optically invisible component (ne; $\sim 55 \%$ ). The radio continuum emission consistently indicates a comparable starburst strength in both components. Assuming that none is substantially contaminated by an obscured active galactic nucleus, and given the high SFR of $500 \pm 250 M_{\odot} \mathrm{yr}^{-1}$, this provides supporting evidence for a ULIRG-like $\alpha_{\mathrm{CO}}$ in both components. The $\mathrm{CO}(J=$ $1 \rightarrow 0$ ) emission in J123707+6214 likely arises from the same gas phase detected in the higher- $J$ lines, but the $\mathrm{CO}(J=1 \rightarrow 0)$ emission appears somewhat more spatially extended. This yields a revised, $\sim 2 \times$ higher estimate for the dynamical mass of the system. Also, this finding would be consistent with the presence of some diffuse, low-excitation gas (which may have a higher $\alpha_{\mathrm{CO}}$ than the highly excited gas). Such a low-excitation component could be associated with gas that is redistributed by mechanical energy input from the starburst or with tidal structure in the ongoing, gas-rich merger in this system.

Our findings highlight the importance of observing multiple $\mathrm{CO}$ lines including $\mathrm{CO}(J=1 \rightarrow 0)$ to determine the total molecular gas mass and gas properties in SMGs (as already acknowledged by Tacconi et al. 2008 in the initial observations of this source). Our results are consistent with those found for other SMGs observed in $\mathrm{CO}(J=1 \rightarrow 0)$ emission (Hainline et al. 2006; Carilli et al. 2010; Ivison et al. 2010; Harris et al. 2010), which commonly show lower CO line excitation than typically found in FIR-luminous quasar host galaxies at comparable redshifts and with comparable gas masses (e.g., Riechers et al. 2006, 2009; Weiß et al. 2007). This provides supporting evidence that both populations trace different evolutionary stages of the same massive galaxy population, as would be expected in the ULIRG-quasar transition scenario proposed by Sanders et al. (1988).

$\mathrm{J} 123707+6214$ is a prototypical example of an SMG during an early merger stage, found in the peak epoch of galaxy formation. Higher resolution, dynamical mapping of $\operatorname{CO}(J=$
$1 \rightarrow 0$ ) emission in this intriguing system (and others) is desirable to narrow down $\alpha_{\mathrm{CO}}$ through dynamical mass measurements over several resolution elements, as possible with the full EVLA in the future. A more complete census of $\mathrm{CO}(J=1 \rightarrow 0)$ observations of SMGs will provide the necessary context to interpret the results of such investigations. Such studies provide the most direct means to constrain the gas fraction, total mass, and evolutionary state of SMGs, which is necessary to better understand the evolutionary path of massive galaxies through their most active phases and to constrain the molecular gas mass density of the universe.

We thank the referee for a critical reading of the manuscript and for a helpful report. D.R. acknowledges support from NASA through Hubble Fellowship grant HST-HF-51235.01 awarded by STScI, operated by AURA for NASA, under contract NAS 526555. The National Radio Astronomy Observatory is a facility of the National Science Foundation operated under cooperative agreement by Associated Universities, Inc.

\section{REFERENCES}

Aravena, M., et al. 2010, ApJ, 718, 177

Blain, A. W., Smail, I., Ivison, R. J., Kneib, J.-P., \& Frayer, D. T. 2002, Phys. Rep., 369, 111

Borys, C., Chapman, S., Halpern, M., \& Scott, D. 2003, MNRAS, 344, 385

Carilli, C. L., et al. 2010, ApJ, 714, 1407

Daddi, E., et al. 2010, ApJ, 713, 686

Dannerbauer, H., Daddi, E., Riechers, D., Walter, F., Carilli, C. L., Dickinson, M., Elbaz, D., \& Morrison, G. E. 2009, ApJ, 698, L178

Downes, D., \& Solomon, P. M. 1998, ApJ, 507, 615

Giavalisco, M., et al. 2004, ApJ, 600, L93

Greve, T. R., et al. 2005, MNRAS, 359, 1165

Hainline, L. J., Blain, A. W., Greve, T. R., Chapman, S. C., Smail, I., \& Ivison, R. J. 2006, ApJ, 650, 614

Harris, A. I., Baker, A. J., Zonak, S. G., Sharon, C. E., Genzel, R., Rauch, K., Watts, G., \& Creager, R. 2010, ApJ, 723, 1139

Ivison, R. J., Papadopoulos, P. P., Smail, I., Greve, T. R., Thomson, A. P., Xilouris, E. M., \& Chapman, S. C. 2011, MNRAS, 412, 1913

Ivison, R. J., Smail, I., Papadopoulos, P. P., Wold, I., Richard, J., Swinbank, A. M., Kneib, J.-P., \& Owen, F. N. 2010, MNRAS, 404, 198

Morrison, G. E., Owen, F. N., Dickinson, M., Ivison, R. J., \& Ibar, E. 2010, ApJS, 188,178

Riechers, D. A., Walter, F., Carilli, C. L., \& Lewis, G. F. 2009, ApJ, 690, 463

Riechers, D. A., et al. 2006, ApJ, 650, 604

Riechers, D. A., et al. 2010, ApJ, 720, L131

Sanders, D. B., Soifer, B. T., Elias, J. H., Madore, B. F., Matthews, K., Neugebauer, G., \& Scoville, N. Z. 1988, ApJ, 325, 74

Solomon, P. M., \& Vanden Bout, P. A. 2005, ARA\&A, 43, 677

Spergel, D. N., et al. 2003, ApJS, 148, 175

Spergel, D. N., et al. 2007, ApJS, 170, 377

Swinbank, A. M., Smail, I., Chapman, S. C., Blain, A. W., Ivison, R. J., \& Keel, W. C. 2004, ApJ, 617, 64

Tacconi, L. J., et al. 2006, ApJ, 640, 228

Tacconi, L. J., et al. 2008, ApJ, 680, 246

Weiß, A., Downes, D., Neri, R., Walter, F., Henkel, C., Wilner, D. J., Wagg, J., \& Wiklind, T. 2007, A\&A, 467, 955

Weiß, A., Downes, D., Walter, F., \& Henkel, C. 2005, A\&A, 440, L45 